\title{
Pregnancy among women undergoing chronic hemodialysis: Effect of ultrafiltration during gestation.
}

\author{
Luiz Paulo José Marques
}

Department of Medicine, Renal Unit of Gaffrèe and Guinle University Hospital, Federal University of Rio de Janeiro State, Rio de Janeiro, Brazil.

Accepted on October 18th, 2018

\section{Short Commentary}

The pregnancy is told as a rare event among End-stage Renal Disease (ESRD) women undergoing chronic Hemodialysis (HD). This is mainly due to ESRD to be considered per se as a powerful method of contraception and sexual dysfunction is higher in women undergoing HD likely as a result of the cumulative effects of depression, fatigue, altered body image, medication side effects and premature menopause [1], and the gestation in these women is frequently associated with poor fetal outcomes.

However, in last years the number of gestations among patients undergoing HD is increasing exponentially around the world, associated with better fetal outcome secondary to improvement in dialysis management during gestation. A systematic review and meta-analysis in pregnancy on dialysis patients regarding the period between 2000 and 2008 collected series of at least 5 cases included 90 pregnancies and when the period was increased in 6 years (from 2000 to 2014) more than 600 pregnancies were included, probably related to the great progress that has been made in the care of HD patients with widespread use of biocompatible membranes, greater dialysis dose and erythropoiesis stimulating agents [2].

Hou was first to demonstrate a significant improvement in live births in women on HD receiving greater than $20 \mathrm{~h}$ of dialysis per week during pregnancy [3]. A significant relationship between dialysis dose and the live birth rate has been demonstrated, improving from $48 \%$ in weekly HD dose $\leq 20 \mathrm{~h}$ to $85 \%$ in HD dose $\geq 37 \mathrm{~h}$. Thus, intensive HD regimens with more cumulative hours on dialysis ( $>20 \mathrm{~h} /$ week) and dialyzing more often ( $>4$ days/week) during pregnancy plays a critical role to promote better control of uraemia, anemia and blood pressure, smaller interdialytic weight gain, less amount of fluid withdraw by UF with fewer episodes of intradialytic hypotension which results in fetal outcome improvement and decreased maternal gestational complications such as preeclampsia and polyhydramnios and has become a standard practice [4].

We study the effect of Ultrafiltration (UF) rate during HD in placental-fetal blood flow [5] by obstetric doppler ultrasonography done in the HD room at the beginning and the end of HD session, in order to obtain doppler velocimetric parameters (pulsatility index and resistance index) of uterine, umbilical and middle cerebral artery: these velocimetric parameters are used to study fetal response to hypoxia in high risk pregnancy.
The dry weight is very difficult to determine during pregnancy as well as to make sure that pregnant woman on dialysis is euvolemic. The expected weight gain in the first trimesters is minimal $(0.5-1.5 \mathrm{Kg} /$ trimester $)$, while from the $24^{\text {th }}$ week of gestation weight gain recommended is up to $0.5 \mathrm{~kg} /$ week and $11.5-16 \mathrm{~kg}$ during the pregnancy for women at their ideal body weight. However, most of weight gain in interdialytic period is of fluid and, arterial hypertension secondary to hypervolemia must be avoided during gestation.

However, it is well known that UF isolated or during HD with removal of significant amounts of fluid can result in circulatory stress and hypotension, associated with poor tissue perfusion of various body tissues and also increase incidence of thrombosis [6]. In pregnant women UF can increase the episodes of intradialytic hypotension which may lead to placental ischemic injury and predispose to fetal distress.

In our hospital cumulative hours on HD during pregnancy is increased to 24-30 h/week with 6 sessions/week (from Monday to Saturday). HD is performed with normal heparin anticoagulation to offer a proper dialysis using high biocompatibility dialyzer with less filtration area to provide lower UF fluid loss and high efficiency (Kt/v from 1.5-1.7, recommended $\mathrm{Kt} / \mathrm{v}>1.2$ ) and with adequate dialysate for gestation (the dialysate is adapted to potassium levels of 3-3.5 $\mathrm{mmol} / \mathrm{L}$, calcium of $1.5 \mathrm{mmol} / \mathrm{L}$ and bicarbonate concentration of $25 \mathrm{mEq} / \mathrm{L})$.

Pregnant patient dry weight is re-evaluated and defined in all first dialysis of the week after dialysis day break in order to avoid unnecessary fluid withdrawn by UF. And when the increase amount of UF rate is required, the time of HD session is enlarged to five hours in order to reduce UF rate and circulatory stress. UF rate is a modifiable fetal risk factor, and increase risk is related to higher UF rate that leads to significant micro vascular blood flow decrease at the end of HD session; this repeated event during pregnancy can result in placental chronic ischemic lesions that may predispose to prematurity and low birth weight.

The goal is to perform HD without UF during pregnancy. However, it is difficult to obtain, even with intensive HD, once most of weight gain in interdialytic period is of fluid. In our study the patient performed to 122 HD sessions after admission in our renal unit and UF was necessary in all HD session [5]. The acute effect of UF in placental-fetal blood flow was studied when UF volume was $\geq 2000 \mathrm{~mL}(2.18 \pm 0.25 \mathrm{~L}) / \mathrm{HD}$ session with UF rate $6 \sim 8 \mathrm{~mL} / \mathrm{h} / \mathrm{kg}$ and doppler velocimetric parameters (pulsatility index and resistance index) before and 
Citation: Marques LPJ. Pregnancy among women undergoing chronic hemodialysis: Effect of ultrafiltration during gestation. J Clin Nephrol Ther 2019;3(1):1-2.

after UF in this gestational period were compared with reference value in normal pregnancy $\left(5^{\text {th }}, 50^{\text {th }}\right.$ and $90^{\text {th }}$ percentile) to the same gestational age and we demonstrated that UF rate: $6 \sim 8 \mathrm{~mL} / \mathrm{h} / \mathrm{kg}$ did not bring any acute harmful effect on placental-fetal blood flow.

The chronic effects of UF during pregnancy on fetal well-being was studied in $122 \mathrm{HD}$ sessions with UF volume withdrawn of $0.5-2.5 \mathrm{~L}(1.4 \pm 0.4 \mathrm{~L}) / \mathrm{HD}$ session with $\mathrm{UF}$ rate $<6 \mathrm{~mL} / \mathrm{h} / \mathrm{kg}$ in $93.5 \%$ of sessions. No chronic fetal deleterious effect was observed, since fetal growth matched to her gestational age and umbilical/middle cerebral artery index was always $<1$. The patient has had a healthy boy by vaginal delivered at $36-37^{\text {th }}$ week and fetal growth matched his gestational age (weight $=2595 \mathrm{~g}$ and height $=47 \mathrm{~cm}$ with Apgar score=9) and they were discharged a few days after delivery. However, we could not rule out that UF during HD may have contributed to prematurity [5].

Pregnancy is possible among women undergoing chronic HD and obstetric and nephrological medical care must be beginning before the conception. Intensive HD regimen with adequate dialysate during gestation plays a critical role to promote better control of milieu uremic, anaemia, maternal volemia and better fetal outcome. UF during HD is a modifiable fetal risk factor and higher UF rate may lead to placental ischemic injury and predispose to fetal distress. When UF is prescribed during gestation it should be individualized, done cautiously and with lower rate as possible in order to avoid acute or chronic fetal deleterious effect.

\section{References}

1. Strippoli GFM. Sexual Dysfunction in Women with ESRD Requiring Hemodialysis. Clin J Am Soc Nephrol. 2012; 7(6): 974-81.
2. Piccoli GB, Minelli F, Versino E, et al. Pregnancy in dialysis patients in the new millennium: a systematic review and meta-regression analysis correlating dialysis schedules and pregnancy outcomes. Nephrol Dial Transplant. 2016; 31(11): 1915-34.

3. Hou S. Daily dialysis in pregnancy. Hemodialysis Int. 2004; 8(2): 167-171.

4. Hladunewich MA, Hou S, Odutayo A, et al. Intensive hemodialysis associates with improved pregnancy outcomes: A Canadian and United States cohort comparison. J Am Soc Nephrol. 2014; 25(5): 1103-9.

5. Marques LPJ, Marinho PRS, Rocco R, et al. Effect of ultrafiltration on placental-fetal blood flow in pregnancy of woman undergoing chronic hemodialysis. Hemodial Int. 2018; 22(3): 405-12.

6. McIntyre C, Crowley L. Dying to feel better: the central role of dialysis-induced tissue hypoxia. Clin J Am Soc Nephrol. 2016; 11(4): 549-51.

\section{*Correspondence to}

Luiz Paulo José Marques

Universidade Federal do Estado do Rio de Janeiro, Brazil.

Rua Conde de Bonfim, 370/502

Postal Code: 20520-054/Tijuca - Rio de Janeiro- RJ./BRAZIL

Telephone: (21) 98208-6626

E-mail: marqueslpj2016@gmail.com 